\title{
Giant Metastatic Breast Phyllodes Tumour with an Elusive Diagnosis: A Case Report and Literature Review
}

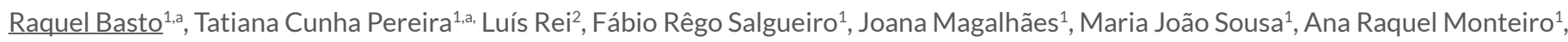 \\ Filipa Macedo ${ }^{1}$, Rita Félix Soares ${ }^{1}$, Teresa Carvalho ${ }^{1}$, Paula Jacinto ${ }^{1}$, Gabriela Sousa ${ }^{1}$ \\ ${ }^{1}$ Medical Oncology Department, Portuguese Oncology Institute of Coimbra Francisco Gentil, Coimbra, Portugal \\ ${ }^{2}$ Anatomic Pathology Department, Portuguese Oncology Institute of Coimbra Francisco Gentil, Coimbra, Portugal

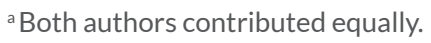

\section{Doi: 10.12890/2021_002763 - European Journal of Case Reports in Internal Medicine - @ EFIM 2021}

Received: 07/07/2021

Accepted: $18 / 07 / 2021$

Published: $23 / 08 / 2021$

How to cite this article: Basto R, Cunha Pereira T, Rei L, Rego Salgueiro F, Magalhães J, Sousa MJ, Monteiro AR, Macedo F, Soares RF, Carvalho T, Jacinto P, Sousa G. Giant metastatic breast phyllodes tumour with an elusive diagnosis: a case report and literature review. EJCRIM 2021;8: doi:10.12890/2021_002763

Conflicts of Interests: The authors declare there are no competing interests.

This article is licensed under a Commons Attribution Non-Commercial 4.0 License

\section{ABSTRACT}

Background: The term phyllodes tumours, which account for less than $1 \%$ of breast neoplasms, describes a spectrum of heterogenous tumours with different clinical behaviours. Less than $30 \%$ present as metastatic disease. Complete surgical resection is the standard of care so that recurrence rates are reduced. The role of adjuvant chemotherapy or radiation therapy is controversial. Patients with metastatic disease have a median overall survival of around 30 months.

Case description: The authors present the case of a 57-year-old woman with an exuberant left malignant phyllodes tumour with bilateral involvement, as well as lung and axillar metastasis. The patient underwent haemostatic radiation therapy and started palliative chemotherapy with doxorubicin, achieving partial response with significant improvement in quality of life. A posterior simple mastectomy revealed a small residual tumour.

Discussion: Metastatic malignant phyllodes tumours are rare, so therapeutic strategies rely on small retrospective studies and guidelines for soft tissue sarcoma. Palliative chemotherapy protocols include anthracycline-based regimens, either as monotherapy with doxorubicin or doxorubicin together with ifosfamide. With few treatment options, management of these patients must rely on a continuum of care.

\section{LEARNING POINTS}

- Phyllodes tumours are a rare type of breast neoplasm.

- The differential diagnosis of breast cancer should include phyllodes tumours.

- Accurate and rapid diagnosis is required

\section{KEYWORDS}

Phylloid, tumor, breast, cancer

\section{CASE DESCRIPTION}

A 57-year-old woman, without a relevant medical history, noticed a $2 \mathrm{~cm}$ wide lump in her left breast in early 2017 but was not concerned. The breast mass progressively increased until August 2019, when she presented to an emergency department for uncontrolled pain and accepted medical care. 
The patient underwent diagnostic investigation, including a left breast CT-guided biopsy in September 2019 with histological results compatible with a mesenchymal tumour. A thoraco-abdominopelvic CT scan revealed full occupation of the left breast area by a large tumoural formation measuring $258 \mathrm{~mm}$ at its widest diameter with a mostly liquid component with peripheral areas of tumour heterogeneity (Fig. 1). In addition to two left enlarged axillary lymph nodes about $1 \mathrm{~cm}$ in diameter, at pulmonary level there were several bilateral metastatic nodules, the largest being $41 \mathrm{~mm}$ in diameter (Fig. 2).

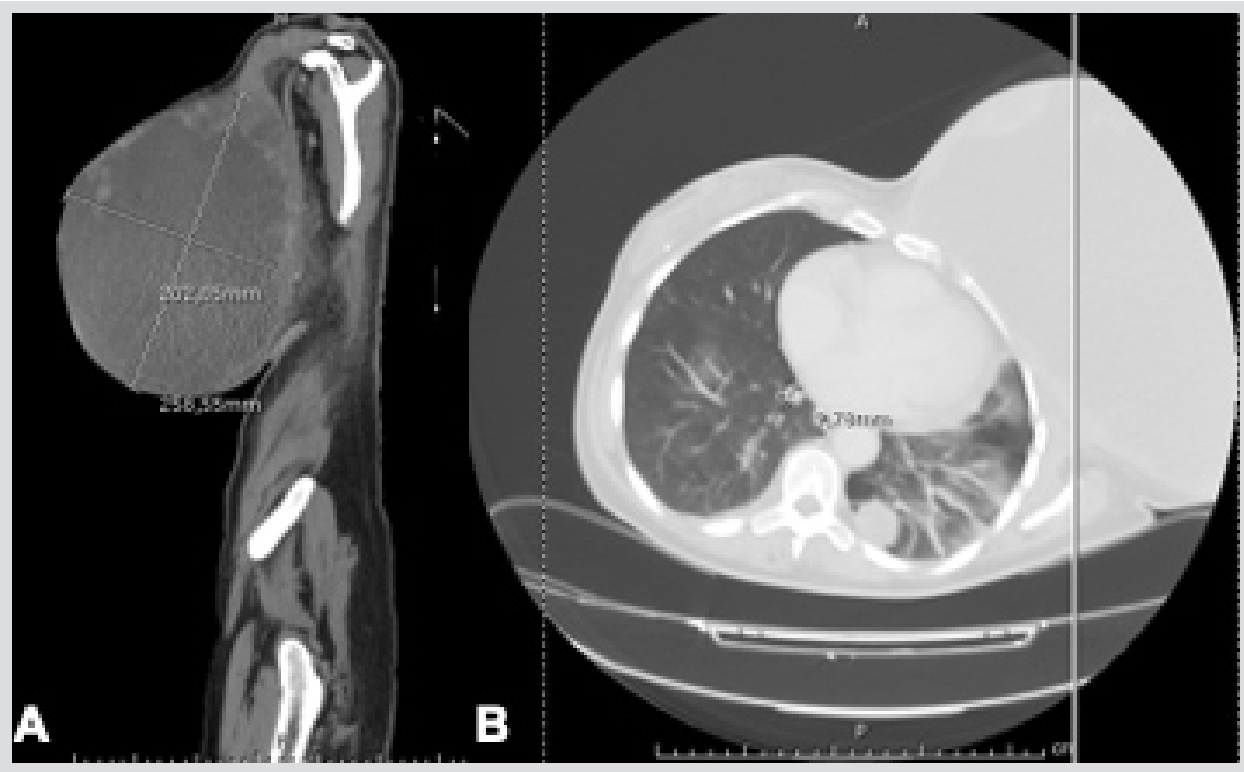

Figure 1. Initial CT scan showing a heterogenous mass: (A) sagittal plane; (B) transverse plane

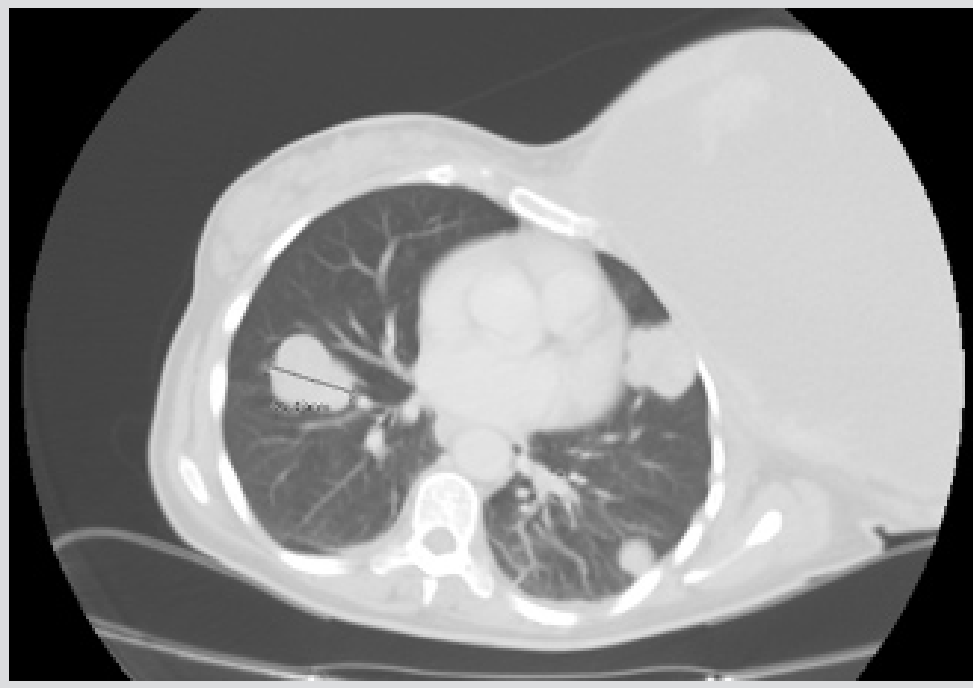

Figure 2. CT scan revealing lung metastases, the largest $41 \mathrm{~mm}$ in diameter

A lung biopsy (Fig. 3) was performed, as well as a confirmatory breast biopsy. Tissue samples were negative for epithelial markers (AE1/AE3, cytokeratin 5/6, cytokeratin 19), P63, oestrogen receptors, S100 protein and smooth muscle actin, but strongly positive for vimentin, so the histological report was compatible with a malignant phyllodes tumour with lung metastasis.

At her first medical oncology appointment in November 2019, the patient had an Eastern Cooperative Oncology Group (ECOG) Performance Status of 0 . She presented with a large, hard exophytic mass measuring $35 \mathrm{~cm}$ at its widest diameter, with three large ulcerative bleeding lesions (Fig. 4), the biggest having a depth of $8 \mathrm{~cm}$ and smelling of putrefaction. Right breast examination revealed several small nodules. Blood work-up revealed grade 4 anaemia (haemoglobin level $5.7 \mathrm{~g} / \mathrm{dl}$ ). Tumour markers were within the normal range: CEA $0.82 \mathrm{ng} / \mathrm{ml}$ (normal range $<3.4 \mathrm{ng} / \mathrm{ml}$ ) and CA $15.3-22.1 \mathrm{U} / \mathrm{ml}$ (normal range $<35 \mathrm{U} / \mathrm{ml}$ ). 


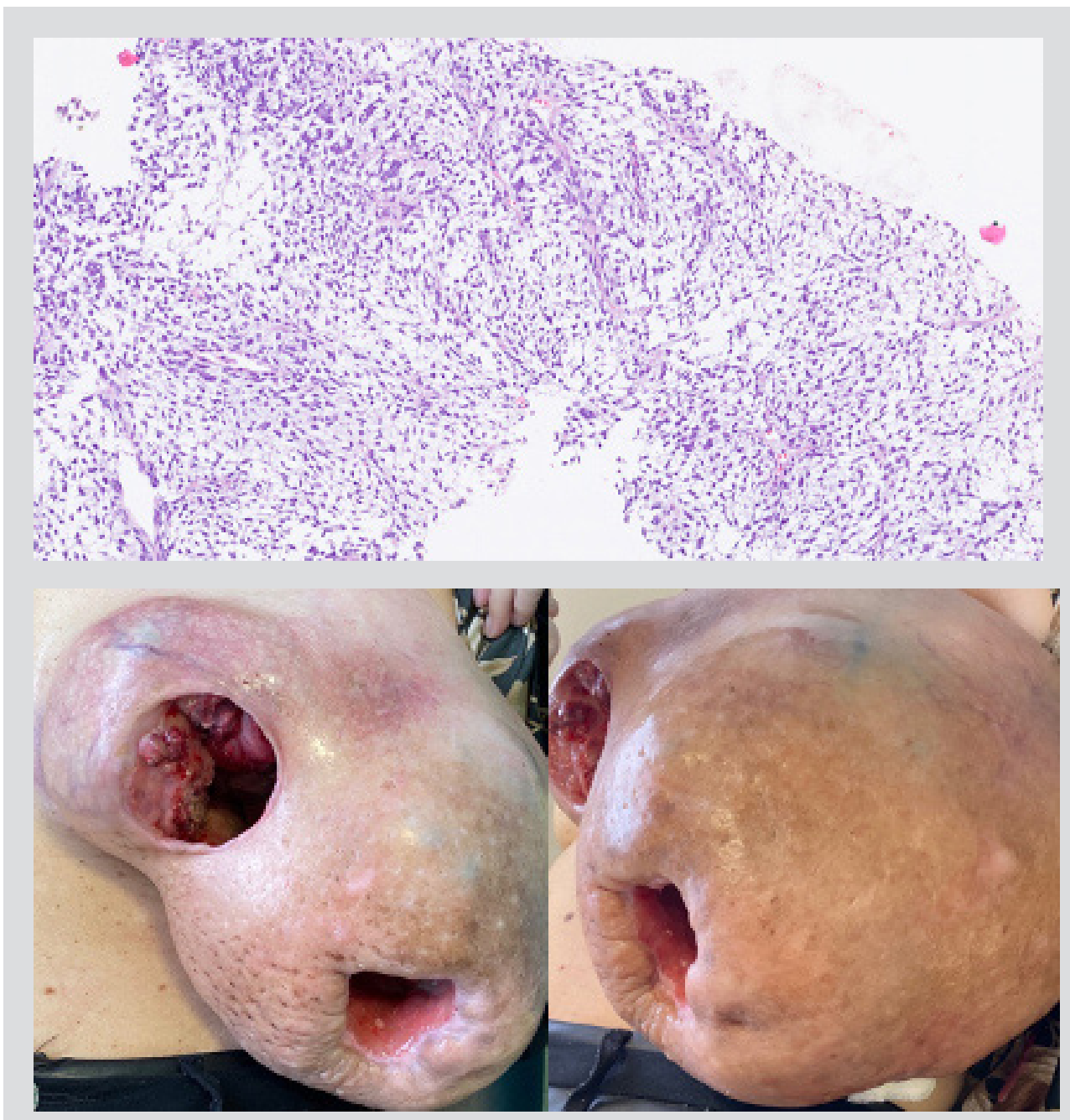

Figure 3. Haematoxylin and eosin staining of a lung biopsy specimen showing fusiform cells within myxoid stroma, as well as moderate cytological atypia with rare mitoses

Figure 4. Large exophytic lesion occupying the entire left breast area with three ulcerative lesions

The patient was admitted to the oncology ward after blood transfusion support with 2 units of packed red blood cells. On the second day of her hospital stay, she started haemostatic radiotherapy (13 Gy/2 fractions) with a progressive decrease in blood transfusion support needs. As a result, she was able to start first-line palliative chemotherapy with doxorubicin at a dose of $70 \mathrm{mg} / \mathrm{m}^{2}$ on 9 December. She was discharged after 16 days of hospitalization and continued chemotherapy in an outpatient clinic.

Tumour assessment by CT scan was performed after four cycles and revealed a heterogenous response, with a slight reduction in the primary tumour with increased gaseous component, but with progression of lung metastasis, the largest nodule being $9 \mathrm{~cm}$ in diameter, with bilateral pleural effusion (Fig. 5).

In view of the local response, the patient continued with doxorubicin for two more cycles with a cumulative dose of $420 \mathrm{mg} / \mathrm{m}^{2}$. Throughout the course of treatment, she reported grade 1 nausea, vomiting and mucositis, as well as grade 1 anaemia, without further need for blood transfusions.

Tumour assessment after six cycles of chemotherapy revealed a partial response of the primary tumour and lung lesions.

Since April 2020, the patient has been under active surveillance and the breast lesion has been regressing, with one of the three initial ulcerative lesions fully resolved.

As a CT scan in July 2020 showed steady disease with the left breast mass measuring $29 \mathrm{~cm}$, a left mastectomy was proposed to the patient in order to improve her quality of life. A simple left mastectomy was performed in September 2020 with removal of a $1714 \mathrm{~g}$ mass measuring $19 \times 17 \times 10 \mathrm{~cm}$. The histopathology report revealed a heterogenous lesion with an area of malignant lesion measuring only $1 \mathrm{~cm}$ at its largest diameter and $2 \mathrm{~cm}$ from the surgical margin, with an epithelial component surrounded by abundant fibrotic tissue. The final diagnosis was compatible with a phyllodes tumour (Fig. 6). 


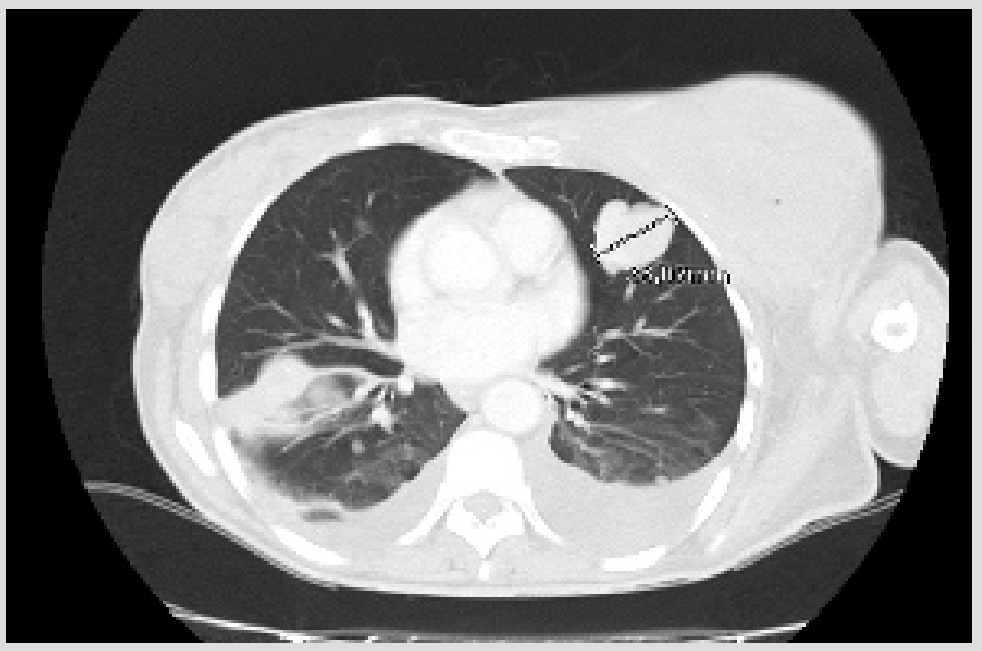

Figure 5. CT scan after four cycles of chemotherapy showing lung disease progression

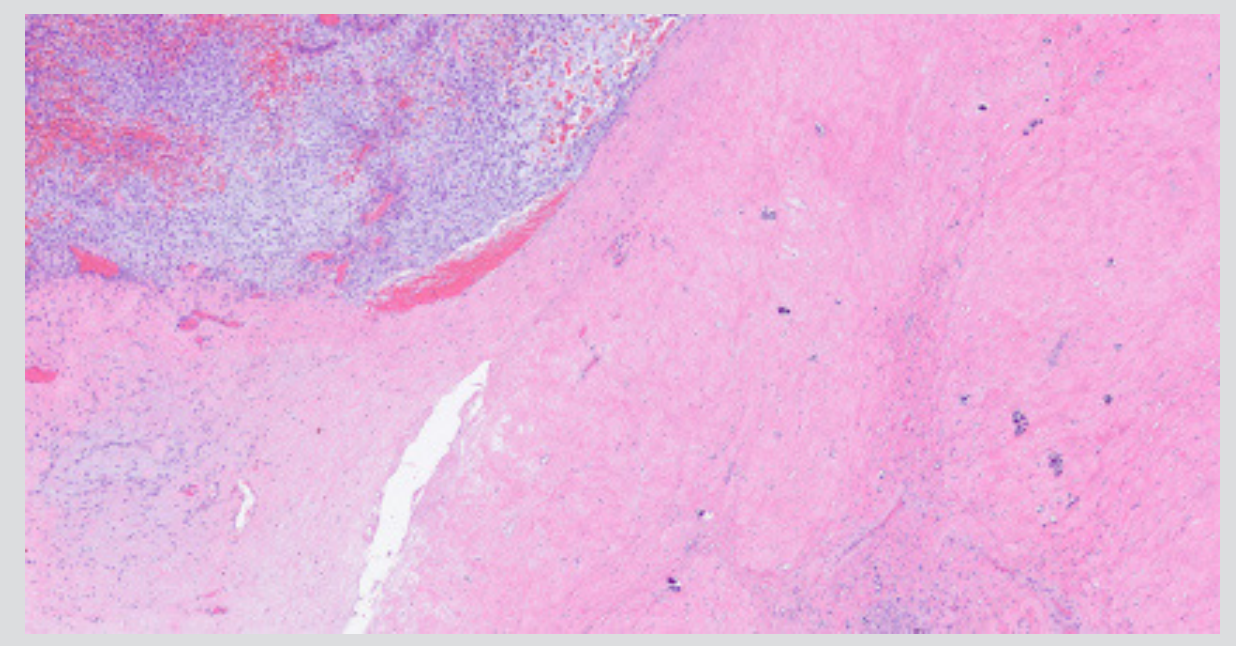

Figure 6. Haematoxylin and eosin staining of a tumour fragment from mastectomy tissue with an epithelial component

\section{DISCUSSION}

Phyllodes tumours are a heterogeneous group of fibroepithelial tumours with diverse biological behaviours, and range from benign fibroadenomas (around 50\%) to malignant invasive phyllodes tumours (less than 30\%) that can degenerate histologically into sarcomatous masses ${ }^{[4,10,11]}$.

Considering its distinct biological behaviour, histologically, phyllodes tumours can be classified as benign, borderline or malignant based upon four characteristics: the degree of stromal cellular atypia, mitotic activity, infiltrative or circumscribed tumour margins and stromal overgrowth. The last feature is the most strongly related to metastatic behaviour ${ }^{[1,12,13}$.

Complete surgical excision is the standard of care for phyllodes tumours in order to reduce local recurrence rates. Spitaleri et al. conducted a multivariate analysis with 172 patients with phyllodes tumours and concluded that a positive margin (despite lack of its definition) is associated with an almost fourfold higher risk of a tumour-related event, such as local recurrence or distant disease (hazard ratio 3.9, 95\% $\mathrm{Cl}$ $1.2-14.3)^{[6]}$. However, new evidence suggests that a positive surgical margin is a concern mostly in malignant phyllodes tumours, as a recent meta-analysis of 54 retrospective studies with 9234 patients found a significantly increased risk of local recurrence only for malignant phyllodes tumours ${ }^{[14]}$. Consequently, there is ongoing debate about management approaches to a positive surgical margin. Moreover, if adequate margins can be achieved, breast-conserving surgery and mastectomy are both effective options for borderline and malignant tumours regarding disease-free survival ${ }^{[15]}$ and cause-specific survival irrespective of tumour size ${ }^{[16]}$.

Despite surgical outcome, phyllodes tumours recur locally depending on tumour grade, with a meta-analysis reporting local recurrence rates of $8 \%, 13 \%$ and $18 \%$ for benign, borderline and malignant tumours, respectively ${ }^{[14]}$.

Axillary lymph node involvement is rare, even when tumours are malignant, so axillary dissection is not mandatory ${ }^{[16]}$. 
Adjuvant radiotherapy reduces the local recurrence rates of borderline and malignant phyllodes tumours, but does not affect overall or disease-free survival. The effect on local recurrence after mastectomy is less pronounced $(\mathrm{HR} 0.68,95 \% \mathrm{Cl}-0.28$ to 1.64$)$ compared with breast-conserving surgery ${ }^{[8]}$.

The role of adjuvant chemotherapy is controversial, mainly due to the fact that malignant phyllodes tumours have a better prognosis than most high-grade sarcomas of a similar stage and there are no randomized control trials.

Metastatic disease is reported in $13-40 \%$ of patients with phyllodes tumours ${ }^{[1,4]}$ with a median overall survival of 30 months, which is affected by mitotic activity and stromal overgrowth and differentiation into osteosarcomatous or chondrosarcomatous features [11]. Phyllodes tumours metastasize mostly to the lungs and, similarly to soft tissue sarcomas, pulmonary metastases should be resected when technically feasible. The choice of chemotherapy schemes in this setting is based upon treatment guidelines for soft tissue sarcoma. For patients with a good performance status and minimal comorbidity, doxorubicin with or without ifosfamide is the first-line chemotherapy regimen. Combined therapy is mainly prescribed for symptomatic patients with a need for quick tumour shrinkage. Other single-agent chemotherapy schemes include dacarbazine or ifosfamide ${ }^{[11]}$.

The randomized phase III GeDDis trial compared gemcitabine plus docetaxel versus doxorubicin as first-line treatment in previously untreated advanced unresectable or metastatic soft tissue sarcomas ${ }^{[17]}$. This trial showed progression-free survival and overall survival similar to doxorubicin. However, gemcitabine-based chemotherapy is an option for patients with contraindication for anthracycline prescription.

Phyllodes tumours show expression of oestrogen and progesterone receptors, but largely in the epithelial component and not the stromal component, so that hormone therapy is not effective against the main driver of metastatic behaviour ${ }^{[18]}$.

The authors have reported a case of a giant metastatic phyllodes tumour treated with first-line chemotherapy with doxorubicin, a regimen mostly studied for soft tissue sarcomas. Because of severe anaemia at first evaluation, as well as a lack of robust evidence for second-line chemotherapy protocols, our team decided to withhold ifosfamide for first progression and proposed the patient for mastectomy in order to improve her quality of life. Mastectomy ultimately revealed a small breast fibrosarcoma only $1 \mathrm{~cm}$ in width. As previously discussed, there are limited data regarding these types of tumours, with most evidence obtained from small retrospective studies, so that management of these patients must rely on a continuum of care.

\section{REFERENCES}

1. Reinfuss M, Mituś J, Duda K, Stelmach A, Ryś J, Smolak K. The treatment and prognosis of patients with phyllodes tumor of the breast: an analysis of 170 cases. Cancer 1996;77(5):910-916.

2. Barrio AV, Clark BD, Goldberg JI, Hoque LW, Bernik SF, Flynn LW, et al. Clinicopathologic features and long-term outcomes of 293 phyllodes tumors of the breast. Ann Surg Oncol 2007;14(10):2961-2970.

3. Karim RZ, Gerega SK, Yang YH, Spillane A, Carmalt H, Scolyer RA, et al. Phyllodes tumours of the breast: a clinicopathological analysis of 65 cases from a single institution. Breast 2009;18(3):165-170.

4. Calhoun K, Allison KH, Kim JN, Rahbar H, Anderson BO. Phyllodes tumors. In: Harris J, Lippman ME, Morrow M, Osborne KC, editors. Diseases of the breast. Philadelphia: Lippincott Williams and Wilkins; 2014, p. 826-837.

5. Jang JH, Choi MY, Lee SK, Kim S, Kim J, Lee J, et al. Clinicopathologic risk factors for the local recurrence of phyllodes tumors of the breast. Ann Surg Oncol 2012;19(8):26122617.

6. Spitaleri G, Toesca A, Botteri E, Bottiglieri L, Rotmensz N, Boselli S, et al. Breast phyllodes tumor: a review of literature and a single center retrospective series analysis. Crit Rev Oncol Hematol 2013;88(2):427-436.

7. Kapiris I, Nasiri N, A'Hern R, Healy V, Gui GPH. Outcome and predictive factors of local recurrence and distant metastases following primary surgical treatment of high-grade malignant phyllodes tumours of the breast. Eur J Surg Oncol 2001;27(8):723-730.

8. Zeng S, Zhang X, Yang D, Wang X, Ren G. Effects of adjuvant radiotherapy on borderline and malignant phyllodes tumors: a systematic review and meta-analysis. Mol Clin Oncol 2015;3(3):663-671.

9. Morales-Vásquez F, Gonzalez-Angulo AM, Broglio K, Lopez-Basave HN, Gallardo D, Hortobagyi GN, et al. Adjuvant chemotherapy with doxorubicin and dacarbazine has no effect in recurrence-free survival of malignant phyllodes tumors of the breast. Breast $J$ 2007;13(6):551-556.

10. Belkacémi Y, Bousquet G, Marsiglia H, Ray-Coquard I, Magné N, Malard Y, et al. Phyllodes tumor of the breast. Int J Radiat Oncol Biol Phys 2008;70(2):492-500.

11. Papas Y, El Asmar A, Ghandour F, Haij I. Malignant phyllodes tumors of the breast: a comprehensive literature review. Breast J 2020;26(2):240-244.

12. De Roos WK, Kaye P, Dent DM. Factors leading to local recurrence or death after surgical resection of phyllodes tumours of the breast. Br J Surg 1999;86(3):396-399.

13. Fajdić J, Gotovac N, Hrgović Z, Kristek J, Horvat V, Kaufmann M. Phyllodes tumors of the breast - diagnostic and therapeutic dilemmas. Onkologie 2007;30(3):113-118.

14. Lu Y, Chen Y, Zhu L, Cartwright P, Song E, Jacobs L, et al. Local recurrence of benign, borderline, and malignant phyllodes tumors of the breast: a systematic review and metaanalysis. Ann Surg Oncol 2019;26(5):1263-1275.

15. Onkendi EO, Jimenez RE, Spears GM, Harmsen WS, Ballman KV, Hieken TJ. Surgical treatment of borderline and malignant phyllodes tumors: the effect of the extent of resection and tumor characteristics on patient outcome. Ann Surg Oncol 2014;21(10):3304-3309.

16. Macdonald OK, Lee CM, Tward JD, ChappeI CD, Gaffney DK. Malignant phyllodes tumor of the female breast: association of primary therapy with cause-specific survival from the Surveillance, Epidemiology, and End Results (SEER) Program. Cancer 2006;107(9):2127-2133.

17. Seddon B, Strauss SJ, Whelan J, Leahy M, Woll PJ, Cowie F, et al. Gemcitabine and docetaxel versus doxorubicin as first-line treatment in previously untreated advanced unresectable or metastatic soft-tissue sarcomas (GeDDiS): a randomised controlled phase 3 trial. Lancet Oncol 2017;18(10):1397-1410.

18. Telli ML, Horst KC, Guardino AE, Dirbas FM, Carlson RW. Phyllodes tumors of the breast: natural history, diagnosis, and treatment. J Natl Compr Cancer Netw 2007;5(3):324330 . 